\title{
Reply to Bombard and Mighton
}

\author{
Daniele Carrieri $\mathbb{1}^{1} \cdot$ Heidi C. Howard ${ }^{2,3} \cdot$ Angus J. Clarke $\mathbb{C}^{4} \cdot$ Vigdis Stefansdottir ${ }^{5} \cdot$ Martina C. Cornel $\mathbb{D}^{6}$. \\ Carla G. van $\mathrm{El}^{6}$. Francesca Forzano ${ }^{7}$
}

Received: 22 November 2018 / Accepted: 22 November 2018 / Published online: 18 January 2019

(c) European Society of Human Genetics 2019

We thank Bombard and Mighton for their comments and for recognising that our contribution "offer a timely set of evidence-based principles that address a critical gap in practice and policy." We also appreciate that Bombard and Mighton raise important points for which additional elaboration may be needed to ensure that the recommendations are clearly understood by readers.

\section{'Duty' to recontact and inequity}

Bombard and Mighton's first consideration concerns equity. The authors appear to argue that not recommending recontacting as a 'duty' and recommending that each country operationalise recontacting in their own way has the potential to result in inequities both in access to information and in outcomes for patients across European countries. We agree that this is a very important issue, and we have discussed it thoroughly when drafting these ESHG recommendations.

Francesca Forzano

Francesca.Forzano@gstt.nhs.uk

francesca.forzano@nhs.net

Egenis, University of Exeter, Exeter, UK

2 Centre for Research Ethics and Bioethics, Uppsala University, Uppsala, Sweden

3 Society \& Ethics Research, Connecting Science, Wellcome Genome Campus, Cambridge, UK

4 School of Medicine, Cardiff University, Cardiff, UK

5 Landspitali - the National University Hospital of Iceland, Department of Genetics and Molecular Medicine, Reykjavík, Iceland

6 Amsterdam UMC, Vrije Universiteit Amsterdam, Department of Clinical Genetics, Section Community Genetics, Amsterdam Public Health research Institute, Amsterdam, The Netherlands

7 Clinical Genetics Department, Guy's \& St Thomas' NHS Foundation Trust, London, UK
The overarching reason to not have recommended recontacting as a firm "duty" at this time is because we believe it would not be feasible for all contexts and could potentially cause more harm than benefit. Specifically, recommending a duty to recontact: (i) is unlikely to be useful or even possible in countries with legislations which do not allow this course of actions [1]; (ii) is likely to create significant practical and legal issues in countries in which recontacting may be legally feasible, but extremely challenging from a practical point of view, due to lack of resources.

As a background to further understand the context in Europe (as opposed to recommendations made for a single country where health care resources may be more homogeneously regulated and easier to summarise), the ESHG is an international organisation which embraces members from different countries, the majority of whom are Europeans. The aim of the ESHG recommendations is to provide guidance to European countries on scientific and clinical topics and issues pertaining to (medical) genetics. Geographically, Europe currently lists 51 countries, 28 of which are European Union members. Each country has a unique legislation and a unique (genetics) healthcare framework (including resources such as budget, workforce, infrastructure). Indeed, these differences may in some cases lead to different resources being available in different countries. This means that a certain degree of inequity is almost inevitable (although the freedom of movement for healthcare through Europe does attempt to remedy this). Stating that all countries, regardless of the resources available therein, have a duty to recontact in genomics, would potentially force already struggling health care systems to make decisions on priorities that are not in the best interest of their larger public.

Furthermore, as Bombard and Mighton also point out, a recent survey on recontacting practices [1] reported that recontacting is already occurring in Europe, but not systematically, with inequalities within and across countries. We think that the guiding principles in our recommendations represent an important step to start 
addressing these inequalities. Arguably, inequities would exist even if recontacting became a legal duty. Legal duties per se would not necessarily make more resources or clearer protocols appear, which could necessarily re-balance the inequities within or between different countries. On the contrary, the existence of a duty, especially in a framework of scarce resources, might well lead to sanctions against those centres that would not be able to comply. This would ultimately increase the inequity.

Therefore, we opted for more nuanced recommendations, pointing forward in a desirable direction, which might prove to be applicable in the short term and which could serve as a useful tool in the revision or implementation of healthcare provision strategies.

While we recognise that leaving recontact to the discretion of clinicians is imperfect, we think this is the best option at present. These recommendations might be considered to be a first step towards possibly more binding recommendations in the future.

\section{Ethnicity and inequity}

We completely agree that the under-representation of genome sequence information across ethnic groups, and the potential necessity for an increasing scale of variant reinterpretation in these subgroups - with a consequent need for additional recontacting - is a very important one. However, this is potentially a broader issue of social justice and global health such that it is more related to who is getting sequenced in the first place, rather than what a clinic or health care region can afford to, or is able to, or is willing to recontact patients (although we also admit there are overlaps in these issues).

\section{Opportunity costs}

We fully agree that the opportunity costs of recontacting deserves attentive scrutiny, and for this reason we touched on this topic several times. Appropriate HTA studies would inform the relevant stakeholders on the most strategic and effective resource allocation, which will be crucial to implement recontacting strategies.

\section{Clinical and personal utility}

We thank Bombard et al. for very pertinently mentioning the difficulties in defining clinical and personal utility. The contents and thresholds of clinical and personal utility are complex and continue to be debated with no clear consensus [2,3]; these notions clearly merit further and more comprehensive discussions, which were not the focus of our recommendations.

Within the context of clinical genetics, with its particular ethical and familial complexities, and in a scenario of a progressively personalised medicine, rather than proposing an operational definition of clinical and personal utility we contend it would be wiser and more workable to suggest tailoring them to the patient's condition and the family's overall situation, recognising that flexibility of judgement in this context should be granted to the clinician in charge. Indeed, as we proceed, more evidence can and should be gathered on reasonable thresholds for personal and clinical utility given available resources and capacity in the context of the jurisdiction, culture, clinic or family.

We are pleased to have already received these constructive comments from colleagues as this shows the utility of our Recommendations to contribute to the discussion and further the research in this setting. We also hope that they can be helpful in driving implementation of recontacting in genetic clinics.

We expect to revise these recommendations in a few years, by which time we hope that more evidence will be available to inform our future policies.

\section{Compliance with ethical standards}

Conflict of interest The authors declare that they have no conflict of interest.

\section{References}

1. Sirchia F, Carrieri D, Dheensa S, et al. Recontacting or not recontacting? A survey of current practices in clinical genetics centres in Europe. Eur J Hum Genet. 2018;26:946-54.

2. Lesko LJ, Zineh I, Huang S-M. What is clinical utility and why should we care? Clin Pharmacol Ther. 2010;88:729-33.

3. Grosse SD, Khoury MJ. What is the clinical utility of genetic testing? Genet Med. 2006;8:448-50. 